\title{
A multimodal imaging approach to the evaluation of post-traumatic epilepsy
}

\author{
Silvia F. Storti $\cdot$ Emanuela Formaggio $\cdot$ Enrica Franchini $\cdot$ \\ Luigi G. Bongiovanni - Roberto Cerini - Antonio Fiaschi • \\ Christoph M. Michel · Paolo Manganotti
}

Received: 27 July 2011 /Revised: 16 April 2012/Accepted: 17 April 2012/Published online: 17 May 2012

(C) The Author(s) 2012. This article is published with open access at Springerlink.com

\begin{abstract}
Object Electroencephalography-functional magnetic resonance imaging (EEG-fMRI) coregistration and high-density EEG (hdEEG) can be combined to map noninvasively abnormal brain activation elicited by epileptic processes. By combining noninvasive imaging techniques in a multimodal approach, we sought to investigate pathophysiological mechanisms underlying epileptic activity in seven patients with severe traumatic brain injury.

Materials and methods Standard EEG and fMRI data were acquired during a single scanning session. The EEGfMRI data were analyzed using the general linear model and independent component analysis. Source localization of interictal epileptiform discharges (IEDs) was performed using 256-channel hdEEG. Blood oxygenation level dependent (BOLD) localizations were then compared to EEG source reconstruction.
\end{abstract}

S. F. Storti $(\bowtie) \cdot$ E. Franchini · L. G. Bongiovanni ·

A. Fiaschi - P. Manganotti

Department of Neurological, Neuropsychological,

Morphological and Movement Sciences, Section of Clinical

Neurology, University of Verona, Policlinico G.B. Rossi,

P.le L.A. Scuro 10, 37134 Verona, Italy

e-mail: silviafrancesca.storti@univr.it

E. Formaggio - A. Fiaschi · P. Manganotti

Department of Neurophysiology, IRCCS San Camillo,

Venice, Italy

R. Cerini

Department of Pathology and Diagnostics, University of Verona,

Verona, Italy

C. M. Michel

Functional Brain Mapping Laboratory,

Department of Fundamental Neurosciences, University Medical

School, University of Geneva, Geneva, Switzerland
Results On hdEEG, focal source localization was detected in all seven patients; in six out of seven it was concordant with the expected epileptic activity as defined by EEG data and clinical evaluation; and in four out of seven in whom IEDs were recorded, BOLD signal changes were observed. These activities were partially concordant with the source localization.

Conclusion Multimodal integration of EEG-fMRI and hdEEG combining two different methods to localize the same epileptic foci appears to be a promising tool to noninvasively map abnormal brain activation in patients with post-traumatic brain injury.

Keywords Post-traumatic epilepsy · EEG-fMRI · Source localization

\section{Introduction}

Post-traumatic epilepsy, a common complication of traumatic brain injury (TBI), occurs in $15-20 \%$ of patients with severe brain trauma. The risk of epilepsy is higher during the first 2 years after both mild and severe TBI, and it remains elevated for more than 10 years after the injury. Clinical seizures are often unrelated to the diffuse and severe brain lesions. And because neurosurgical treatment is generally unfeasible, correlations between electroencephalography (EEG) activity, clinical seizures and brain lesions are rarely investigated.

The combined use of EEG and functional magnetic resonance imaging (fMRI) techniques in epilepsy allows for the noninvasive hemodynamic characterization of interictal activity. EEG-fMRI recording can provide information on the pathophysiological processes underlying interictal activity, since the hemodynamic changes are a consequence 
of the abnormal neural activity generating interictal epileptiform discharges (IEDs). In detail, fMRI measures the hemodynamic response induced by neural activity and can be used to display the active brain regions associated with a process. fMRI does not directly measure neural activity but rather the changes in oxygenation and blood flow. The advantages of fMRI include high spatial resolution (order of $\mathrm{mm}$ ) and whole brain coverage. Moreover, fMRI combined with standard neurophysiological stimulation techniques can provide important clinical insights into epileptic processes. Conversely, EEG measures with high temporal resolution the neural activity from the scalp generated by brain current sources activity, producing a current dipole moment in each tissue volume [40]. For decades EEG has been used to study and characterize pathophysiologic processes in the brain, but it has a limited ability to localize activity to a deep brain region. Used separately, EEG and fMRI provide low spatial (EEG, order of $\mathrm{cm}$ ) or temporal (fMRI, order of s) resolution, but when combined they have complementary features. Used simultaneously, EEG and fMRI is a powerful and noninvasive method to investigate the spatiotemporal mechanisms of abnormal brain activity and to precisely define the irritative zone. The relationship between neuronal physiology and the observed blood oxygenation level dependent (BOLD) activity is still unclear. Research results so far suggest a predominantly linear coupling between BOLD and neuronal activity [32, 41].

These technologies were combined by [24] and are widely used in studies of epileptic patients to localize epileptogenic foci $[1,4,5,7,12,19,25,26,28-31,33,34$, $42,46]$. The results have been corroborated in several important reviews [37, 45, 47].

The conventional analysis of EEG-fMRI data relies on the general linear model (GLM). The neurologist visually identifies IEDs, and the convolution of these EEG events with a model of the fMRI response, i.e., the hemodynamic response function (HRF), provides the regressor for GLM analysis of fMRI data. The significance in activation is then tested by means of a $t$ test which compares the signal intensity of IEDs with that of resting status. In the absence of a paradigm design, a useful alternative is a multivariate approach/analysis such as independent component analysis (ICA). This important technique of data-driven analysis can be applied to solve the problem of blind source separation of signals by dividing the imaging data into several spatial patterns or independent activation maps. ICA can also extract task-related as well as physiologically-relevant non-task-related components and artifactual components. Although ICA decomposition in fMRI is widely used to identify networks, a gold standard selection criteria to separate the ICs related to an internal network from noiserelated ICs is still lacking. The existing methods only rank the components, after which the neurologist must scroll each component manually. Nonetheless, ICA decomposition is extremely useful for identifying IED-related activity [39] and the resting state networks (RSNs) of neuronal activity as regions involved in motor function, visual and auditory processing, memory, executive functioning and the default mode network (DMN). In order to identify the IED-related ICs, the design matrix constructed with the GLM can be compared with the components by computing the cross correlation between them.

Another commonly employed technique in the evaluation of epilepsy is electrical source imaging (ESI). ESI can estimate the localization within the brain volume of the electric source(s) generating an IED that can be recorded with scalp electrodes. ESI involves numerous scalp electrodes, highdensity EEG (hdEEG), and realistic head models derived from structural MRI. The assumption about the number of electrodes, geometric and anatomical properties, conductive volume and focus characteristics can enhance the precision of the inverse solution [38]. Source localization can be used as part of evaluation of patients with focal epilepsy.

In contrast to standard EEG, hdEEG is a recording method that relies on numerous electrodes to increase spatial sampling. Also, hdEEG can be combined with realistic head models and used in source analysis to determine the generators of IED. To date, few researchers have been able to acquire both signals that could reproducibly demonstrate a correspondence between the EEGfMRI signal and the electrical sources $[56,58]$, given that the generators of the EEG and the BOLD signals do not always completely overlap. A recent review of the combined use of EEG-fMRI coregistration and EEG source analysis for event localization in epilepsy reported promising perspectives for these strategies [57].

In this study, we combined noninvasive imaging techniques in a multimodal approach to investigate the pathophysiological mechanisms of epileptic activity in patients with severe TBI. Here, we describe seven cases of pharmacoresistant post-traumatic epilepsy. Assessment was performed using a multimodal approach with the use of hdEEG and EEG-fMRI technologies. The multimodal approach consists of the source localization applied to 256 EEGchannel data and GLM and ICA analysis of the EEG-fMRI data. Finally, the data were independently analyzed and the results compared by verifying the concordance between EEG source localization and BOLD response to interictal spikes.

\section{Materials and methods}

\section{Patients}

The study sample was seven patients with post-traumatic epilepsy. Clinico-pathological characteristics were roughly 
similar: complex partial seizures (focal) and seizure frequency (from 3 to 25 seizures per month); 5 out of 7 patients presented with cognitive and behavioral impairment; all showed focal activity, spike and slow-wave paroxysms. Paroxysmal activity predominated in the left frontal regions (5/7 patients), in the right-temporal regions $(1 / 7)$ and in the left-central regions (1/7).

Patient No. 1 This 43-year-old man sustained severe TBI in a road accident. The first seizures developed 2 years later, with initial clonic movements of the right arm and hand, followed by right leg and orofacial automatisms of the right side of the face, deflection of the face rightward, and early loss of consciousness. MRI revealed left brain cortical and subcortical atrophy with considerable ex vacuo enlargement of the lateral ventricles. Interictal standard scalp EEG over the right hemisphere was inconstant; low voltage $(8-9 \mathrm{~Hz})$ EEG over the left hemisphere showed theta waves associated with polymorphic delta waves which were more evident on the fronto-central hemisphere electrodes; specifically, high amplitude (75-120 $\mu \mathrm{V}) 2 \mathrm{~Hz}$ spike and wave discharges over the left frontal hemisphere electrodes which spread to the right hemisphere.

Patient No. 2 This patient suffered head trauma at age 16 years, with bleeding in the left fronto-polar and frontal regions. Two years later, the first seizure occurred during the night and was described as grand mal seizures with tonic clonic movements of the upper and lower limbs. These were followed by 2-3 episodes of cyclic clinical tonic-clonic seizures per week. Seizure occurrence eventually ceased with additional therapy. Standard EEG showed theta-wave activity in the left frontal regions with phase reversal in F3. Other seizures were characterized by abrupt loss of consciousness, fixed gaze, facial contraction and mild head version to the right.

Patient No. 3 At the age of 14 years, this patient suffered an accidental fall resulting in extradural hematoma and right hemiparesis which resolved (lasting 3 days). Two apparently generalized tonic-clonic seizures occurred during sleep at age 38 years, in addition to sporadic left lower limb clonic seizures during drowsiness. The patient presented no motor deficit, sensory, cognitive or behavioral impairment. Standard EEG showed slow left fronto-temporal foci (drug-resistant) associated with paroxysmal isolated elements in F7. The patient showed continuous theta activity, sometimes polymorphic delta sequences, of medium voltage on the left frontal electrodes. At the same site, isolated slow peaks were detected mainly in Fp1/F7. Rare and isolated polyspikes associated with left frontal slow waves on derivations were recorded only in stage II NREM sleep. These elements showed phase reversal in F7, without a tendency to spread, and disappeared upon awakening.
Patient No. 4 This patient sustained a fracture of the right cranial vault in a motorcycle accident 12 years earlier. After the accident, he fell into coma for 22 days. The front vault was removed and a prosthesis inserted. MRI findings were principally bilateral frontal lesions. He presented right-sided blindness resulting from trauma-induced lesion of the optic nerve and residual orbitofrontal asymmetry of the facial bones. Interictal standard EEG showed theta polymorphic sequences during drowsiness associated with isolated large-voltage paroxysms on the left frontotemporal electrodes with phase reversal in F7. These elements did not tend to spread contralaterally. His history included brief attacks typically occurring in clusters and often at night, characterized by oscillatory trunk movements, arm flexion, synchronous bi-manual automatisms, and proximal isolated movements of the lower limbs associated with loss of consciousness.

Patient No. 5 This 44-year-old man suffered a head injury in a workplace accident at the age of 31 years, associated with an acute left fronto-temporo-parietal subdural hematoma, multiple brain injuries and cerebral edema resulting in right hemiplegia. The patient also presented with motor aphasia. Simple focal seizures first occurring 9 months after the accident were characterized by initial deviation of the mouth and eyes to the right, followed by clonic movements of the upper and then the lower right limb, lasting less than $1 \mathrm{~min}$, on an average of 1-2 times a year. MRI showed bilateral lesions in the frontal regions and in the left temporo-parietal regions. EEG recordings were characterized by slow theta, sometimes delta, polymorphic activity intermixed with superimposed sharp waves showing phase reversal to $\mathrm{C} 3$.

Patient No. 6 At age 19 years, this patient sustained the first and the most severe head trauma in a road accident, resulting in a left fronto-orbital lesion. Reconstruction of the front and placement of implants were performed. Four years after the accident, the patient experienced two episodes of morpheic seizures. One year later, he sustained another head injury, followed by focal and secondarily generalized seizures (starting with prolonged vocalization and quickly followed by a tonic and then a clonic phase). The last seizure occurred 4 months before the present fMRI study. MRI showed a large left frontal and an orbitofrontal poroencephalic lesion associated with significant gliosis. EEG recordings showed persistent epileptogenic foci, with theta activities intermixed with superimposed spikes and waves in the left frontotemporal regions, with maximum appearance at the F7 electrode.

Patient No. 7 This patient presented bilateral lesions after sustaining a work-related accident (right temporal lesion and left temporal lobe lesion). His clinical picture 
was characterized by temporal seizures with temporoparietal aura characterized by auditory hallucinations ("like hearing the sea inside a seashell") followed by epigastric sensations, nausea and loss of consciousness. $\mathrm{He}$ sometimes experienced complex hallucinations ("like a slide series about my past life") associated with oral automatisms, head version to the right, fixed gaze and frothing at the mouth. The patient showed theta-delta activity with sharp, large-voltage morphology in the frontotemporal region and phase reversal in $\mathrm{T} 4$.

\section{Data acquisition}

\section{HdEEG}

HdEEG recording was performed using 256 channels (Electrical Geodesic, Inc., Eugene, OR, USA). The net was adjusted so that $\mathrm{Fpz}, \mathrm{Cz}, \mathrm{Oz}$ and the pre-auricular points were correctly placed according to the international 10/20 system. Due to the geodesic tension structure of the net all electrodes were evenly distributed on the scalp at approximately the same location across subjects. The data were recorded against a vertex electrode reference $(\mathrm{Cz})$ at a sampling rate of 200 or $250 \mathrm{~Hz}$ and filtered off-line with a band-pass filter $(1-30 \mathrm{~Hz})$.

\section{EEG-fMRI data}

During MRI scanning, EEG data were acquired using an MR-compatible EEG amplifier (SD MRI 32, Micromed, Treviso, Italy) and a cap with $32 \mathrm{Ag} / \mathrm{AgCl}$ electrodes positioned according to a 10/20 system. To remove pulse and movement artifacts during scanning, two of the electrodes were used to record the electrocardiogram (ECG) and electromyogram (EMG). The EMG electrode was placed on the right abductor pollicis brevis muscle. The reference was placed anterior to $\mathrm{Fz}$, and the ground posterior to $\mathrm{Fz}$, as previously described elsewhere $[15,51]$ for the same system. EEG data were acquired at a sampling rate of $1,024 \mathrm{~Hz}$ using the SystemPlus software package (Micromed, Italy). To avoid saturation, the EEG amplifier had a resolution of 22 bits with a range of $\pm 25.6 \mathrm{mV}$. An anti-aliasing hardware band-pass filter was applied with a bandwidth of $0.15-269.5 \mathrm{~Hz}$.

Functional images were acquired on a $1.5 \mathrm{~T}$ MR scanner (Symphony, Siemens, Erlangen, Germany) equipped with echo-planar imaging (EPI) capability and a standard transient/receive (TR) head coil. fMRI data were acquired with a $T 2^{*}$ weighted EPI sequence ( 36 slices, $\mathrm{TR}=3,700 \mathrm{~ms}$, $\mathrm{TE}=50 \mathrm{~ms}, 64 \times 64$ matrix, $\mathrm{FOV}=256 \times 256$, slice thickness $3 \mathrm{~mm}$; voxel size $=3 \times 3 \times 3 \mathrm{~mm}$, axial slice orientation). At the start of each fMRI acquisition, the scanner gave a trigger signal that was recorded by the EEG system and used as a volume marker. A T1-weighted anatomical scan (192 slices, TR $=1,990 \mathrm{~ms}$, TE $=3 \mathrm{~ms}$; scanning matrix $512 \times 512, \mathrm{FOV}=256 \times 256$; slice thickness $1 \mathrm{~mm}$; sagittal slice orientation) was also acquired for each patient.

The patients were positioned supine on a bed inside the scanner bore, with elbows flexed at $120^{\circ}$ and hands pronated in a relaxed position; the head was stabilized on both sides with adjustable padded restraints. During fMRI acquisition, the patients were instructed to keep their eyes closed and remain as still as possible throughout the procedure. A total of 200 volumes were acquired for patient No. 1, and 140 volumes for the other 6 patients; the duration of one volume was $3,700 \mathrm{~ms}$.

\section{Data analysis}

\section{Source localization}

HdEEG data were analyzed using Cartool software (http://sites.google.com/site/cartoolcommunity/). The T1 anatomical images acquired before each EEG-fMRI session were used to create a realistic model of the brain for source localization. The original MRI is anisotropic. The images were converted in isotropic using Brain Voyager (Brain Innovation, Maastricht, The Netherlands) with a voxel size of $1 \times 1 \times 1 \mathrm{~mm}^{3}$. In each patient, the hdEEG and MRI data and the solution space were restricted to the gray matter. The solution space for the distributed source model contains from a min of 3,010 to a max of 3,032 points uniformly distributed over the gray matter of the brain mapped onto the spherical head model with anatomical constraints (SMAC) space [50]. The SMAC head model method has been successfully employed in several previous clinical and experimental studies [9, 22, 38, 49, 58 ] and it produces localization precisions comparable with realistic boundary element models [23]. The EEG electrode positions were matched to the MR scalp surface using transformation operations such as rotation and translation according to common landmarks during both EEG and MRI acquisition. The peak of the spike was used as a trigger for averaging in epochs of $\pm 500 \mathrm{~ms}$. The number of spikes ranged from 35 to 42 . Two time frames were chosen to characterize spike topography: the first, from the beginning of the spike to the time point corresponding to $50 \%$ of the rising phase as an epoch characterizing a source of the possible spike generator [27]; the second, an epoch at the peak of the spike indicating propagation. Special attention was paid to marking the same type of spikes as those obtained during the recordings inside the scanner. A standardized source imaging procedure, low resolution brain electromagnetic tomography (LORETA) [43] constrained to the individual matter, was applied to the averaged spikes. 


\section{GLM on EEG-fMRI data}

EEG pre-processing The data were processed using Matlab 7 (MathWorks, Natick, MA, USA) and EEGLAB 4.51 (http://sccn.ucsd.edu/eeglab/) [14]. The EEG artifact induced by the magnetic field gradient was digitally removed off-line using an adaptive filter [3], while the EEG artifact associated with pulsatile blood flow, ballistocardiogram, was removed using an averaging procedure [2], both of which were implemented with SystemPlus software (Micromed). Reference-free recordings were then obtained by calculating the local average reference using EEGLAB. A notch filter $(50 \mathrm{~Hz})$ was also applied to all channels. EEG recordings were band-pass filtered from 1 to $30 \mathrm{~Hz}$ using a FIR filter because all seven patients had a routine EEG recording showing focal IEDs at frequencies above $1 \mathrm{~Hz}$. The signal was then baseline corrected and detrended; epochs with artifacts were visually identified and excluded from the analysis.

fMRI pre-processing Before statistical analysis, the functional data were pre-processed using Brain Voyager software. The MR images were realigned to reduce the effect of head motion (three-dimensional motion correction with sinc interpolation). To correct for the different acquisition times, slice scan time correction was used in an ascending, interleaved scanning order with linear interpolation over time. The data were then pre-processed with linear trend removal to remove all drifts, and with a temporal high-pass filter (three cycles in time course) to reduce the effect of breathing and physiological noises. The anatomical and functional data were kept in the subject's native space. The optional temporal smoothing filter was not used during analysis, while spatial Gaussian smoothing was used with Gaussian kernel $6 \mathrm{~mm}$ full width at half maximum (FWHM).

GLM analysis The analysis of EEG-fMRI data was based on the visual identification of the IEDs on scalp EEG. The convolution of these EEG events, represented as stick functions, with the HRF provided the regressor for GLM analysis of fMRI data. Activated voxels were identified with a single-subject GLM approach for the time series data [17]. Confounds were included to account for motionrelated effects. Temporal autocorrelations were accounted for by fitting an autoregressive (AR) model. Noise was modeled as an AR process estimated from the residuals by using Yule-Walker equations. The partial autocorrelation plots were examined to identify the AR order. A statistical significance was found for lag 1, indicating that the AR model was appropriate for all patients, except for patient No. 3. The confidence interval for statistical significance was of $95 \%$. Brain activation was detected by comparing the signal intensity of active images (IED activity) with that of resting images based on the changes in local BOLD signals and on the voxel-wise Student's $t$ test (with FDR correction according to the number of voxels related to the brain volume). $Z$-score maps representing brain activation were then generated from $t$-maps. The results were displayed as parametric statistical maps, where the pixel $Z$ value is expressed on a colorimetric scale.

\section{PICA on EEG-fMRI data}

fMRI pre-processing The functional data were pre-processed using Multivariate Exploratory Linear Decomposition into Independent Components (MELODIC) version 3.09, a part of the FSL toolbox (http://www.fmrib. ox.ac.uk/fsl). The images were smoothed with a Gaussian kernel $6 \mathrm{~mm}$ (FWHM) and motion-corrected. To correct for the different acquisition times, an interleaved slice timing correction was used. The data were then pre-processed with high-pass temporal filtering (cut-off of $100 \mathrm{~s}$ ) and with the removal of non-brain structures from the echo planar imaging volumes (Brain Extraction Tool [BET] http://www.fmrib.ox.ac.uk/fsl).

PICA analysis The pre-processed data underwent probabilistic independent component analysis (PICA) decomposition using MELODIC. The maps were thresholded at a posterior probability threshold of $p>0.5$ [6]. A threshold of $z$ scores was used to visualize the IC maps. From a variable number of ICs obtained by PICA decomposition (range 13-36 components), the components were then visually selected by an expert neurologist. In order to identify which components were related to a patient's IED activity, the design matrix constructed in the GLM analysis was fitted to the time course of each component. Components significantly related to IED activity were identified with an $F$ test $(p<0.05$, corrected for the number of components) [39]. Statistically significant $F$ values identify ICs time courses showing significant signal changes timelocked to the IED activity.

\section{Results}

Comparison of ESI with EEG-fMRI results

Clinical information and results for each patient are summarized in Table 1. A homogeneous population was studied to explore methodological issues. This group of post-traumatic epilepsy patients was consistent in its number with some clinico-pathological correlations.

On hdEEG, focal source localization was detected in all patients. In six out of seven patients, it was concordant with 


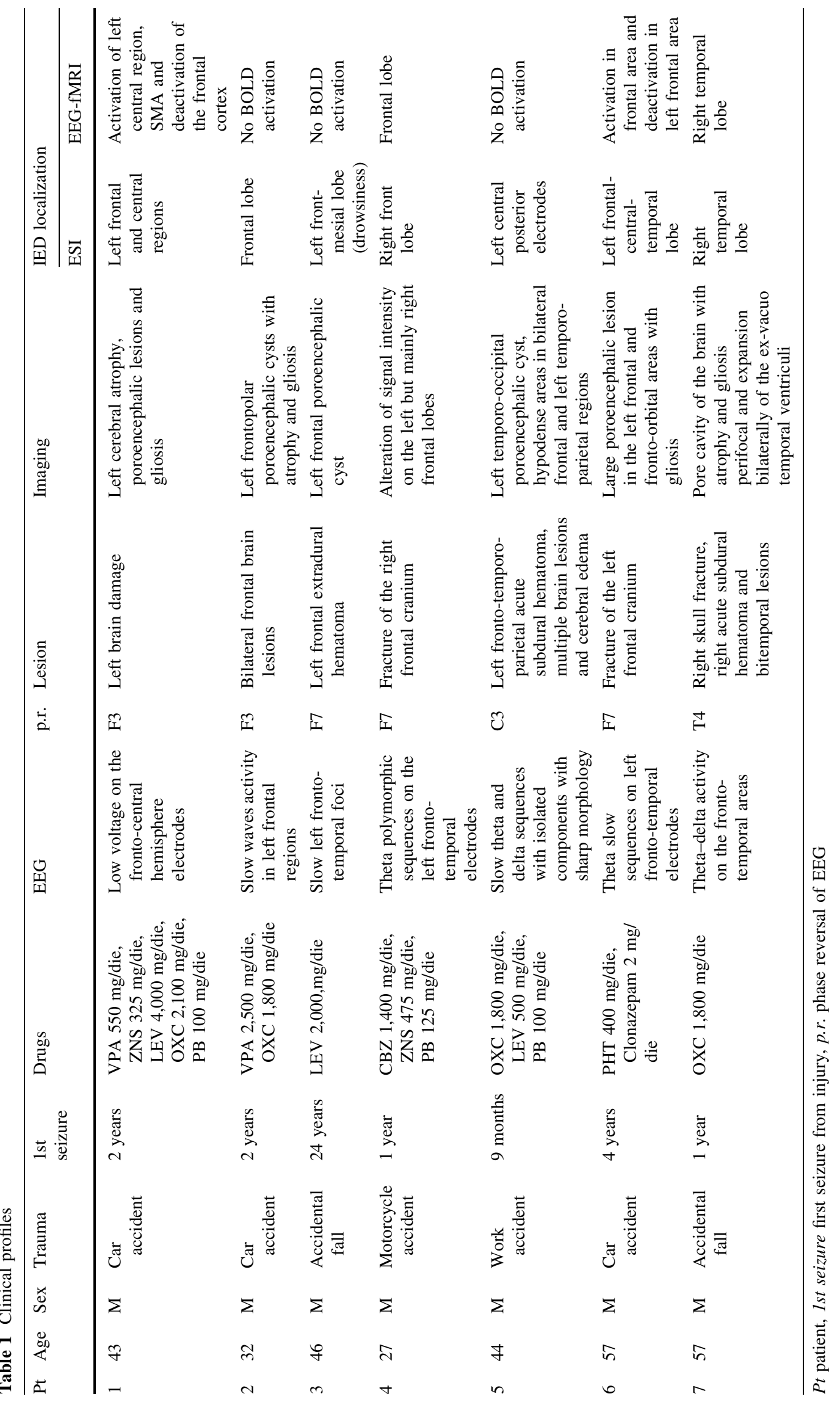


the expected epileptic activity as defined by EEG data and clinical evaluation. BOLD signal changes were observed in four out of seven patients in whom IEDs were recorded (patients Nos. 1, 4, 6 and 7). These activities were concordant with the source localization in patients Nos. 1,6 and 7 and only partially concordant in patient No. 4. In some patients, the IEDs inside the magnet were not very frequent and of limited duration, which subsequently created difficulties in the off-line analysis. ICA identified IEDrelated activity in three out of seven patients (patients Nos. 1, 4, and 6). DMN was not always detected (i.e., patients Nos. 1 and 5).

\section{Single-subjects results}

Patient No. 1 HdEEG activity showed sequences of spike and wave complexes over the left fronto-central regions lasting more than $10 \mathrm{~s}$ (Fig. 1a). The source localization showed a defined localization over the rolandic areas compatible with focal EEG activity (Fig. 1b). EEG activity inside and outside the magnet showed a high amplitude over the left fronto-polar electrodes, with a phase reversal over the frontal and central electrodes (Fig. 1c). The GLM analysis showed prominent BOLD activation over the left central cortical areas (rolandic and sylvian) adjacent to the brain damage and anterior to the ventricular enlargement and over the supplementary motor areas (SMA) during the spikes and wave discharges as compared to the resting state. A deactivation in the left frontal and parietal regions, not observed in the source analysis, was also obtained, FDR $(q<0.05)$ (Fig. 1d). The PICA decomposition estimated 36 components, five of which were related to internal processes. Components nos. 30 and 25 were significantly IED-related ICs: the first was positively correlated with the GLM design matrix and the second negatively ( $F$ test, $p<0.05$, corrected for the number of components); component no. 20 represented the occipital network; components nos. 16 and 17 the lesion-related ICs. The DMN was not detected (Fig. 1e).

Patient No. 2 HdEEG showed slow-wave activity in the perilesional frontal and prefrontal regions, consistent with the head trauma (Fig. 2a). The source localization showed a defined localization over the frontal region (Fig. 2b). The EEG acquired inside the scanner showed predominant left frontal activity (Fig. 2c). The EEG-fMRI activation maps showed no significant activation, probably due to the short duration of sequences characterized by IED activity, FDR $(q<0.05)$ (Fig. 2d). The PICA decomposition estimated 35 components, four of which were related to internal processes: the DMN (component no. 34); the temporal network, a network primarily including the bilateral superior temporal cortex, corresponding to the auditory- phonological system (component no. 30); and two components related to the lesion area (components nos. 2 and 19). No IED-related IC was identified (Fig. 2e).

Patient No. 3 HdEEG data acquisition lasted $40 \mathrm{~min}$; during drowsiness the patient showed activity with a maximal amplitude over the left central and frontal regions (Fig. 3a). The source localization showed left frontal mesial activity (Fig. 3b). The EEG acquired inside the magnet showed predominant activity over the left frontal electrodes, with a phase reversal in F7 (Fig. 3c). No BOLD activity was detected with GLM analysis, FDR $(q<0.05)$ (Fig. 3d). The PICA decomposition estimated 18 components, three of which were related to internal processes: the DMN (component no. 13); the attention network (component no. 16); and the temporal network (component no. 8). No IED-related IC was identified (Fig. 3e).

Patient No. 4 HdEEG showed high activity over the right frontal electrodes, as confirmed by the source analysis (Fig. 4a, b). EEG inside the MR scanner showed high activity on the frontal electrodes (Fig. 4c). The patient underwent two continuous EEG-fMRI sessions. During the first fMRI scanning, a spontaneous critical episode lasting about $41 \mathrm{~s}$ was recorded; it was characterized by arm lifting and flexion, synchronous bi-manual automatisms, and ended with extension and repositioning of the limbs on the chest. Isolated synchronous movements of the lower limbs occurred towards the middle of the critical episode. The first delta rhythmic sequences and later muscle and motion artifacts suddenly appeared in the right frontal lobe. During attenuation of the motor phenomena, delta sequences were recorded in the left frontal regions. The second fMRI scan was recorded during interical events and used for the analysis (Fig. 4c). BOLD activity was perilesional and localized over the frontal area, FDR $(q<0.05)$ (Fig. 4d). The PICA decomposition estimated 22 components, five of which were selected: one related to IED activity (component no. 20); one related to the DMN (component no. 16); one related to the temporal network (component no. 9); and two related to the area of the lesion (components nos. 3 and 14) (Fig. 4e).

Patient No. 5 HdEEG showed high activity over the left central posterior electrodes (Fig. 5a). The source was localized over the left residual rolandic area (Fig. 5b). EEG acquired inside the scanner showed slow activity in the left motor area, with phase reversal in C3 (Fig. 5c). No BOLD activity was detected with GLM analysis, FDR $(q<0.05)$ (Fig. 5d). The PICA decomposition estimated 21 components, two of which related to the area of the lesion (components nos. 11 and 15) were selected. No components related to DMN or IED activity were identified (Fig. 5e). 
a

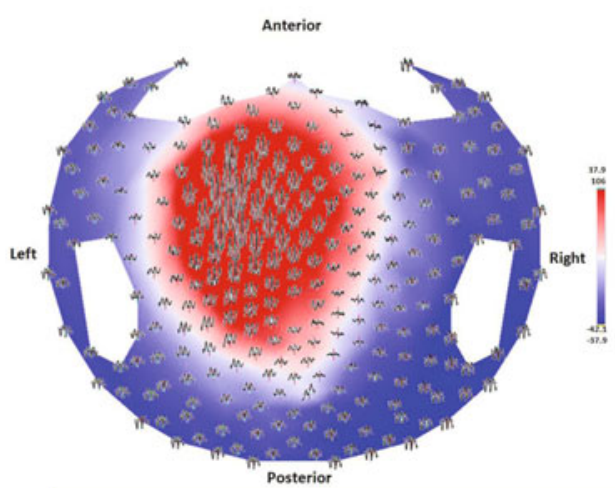

C

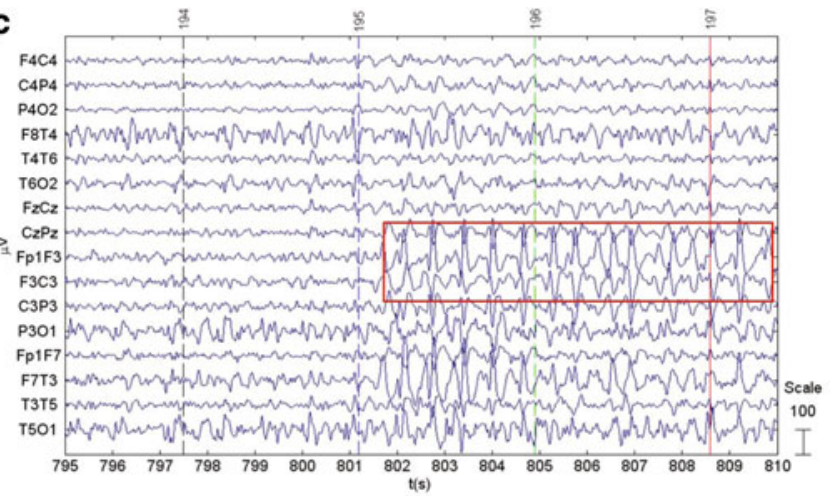

b

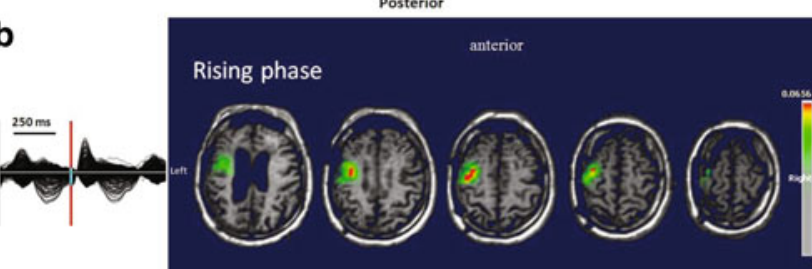

d
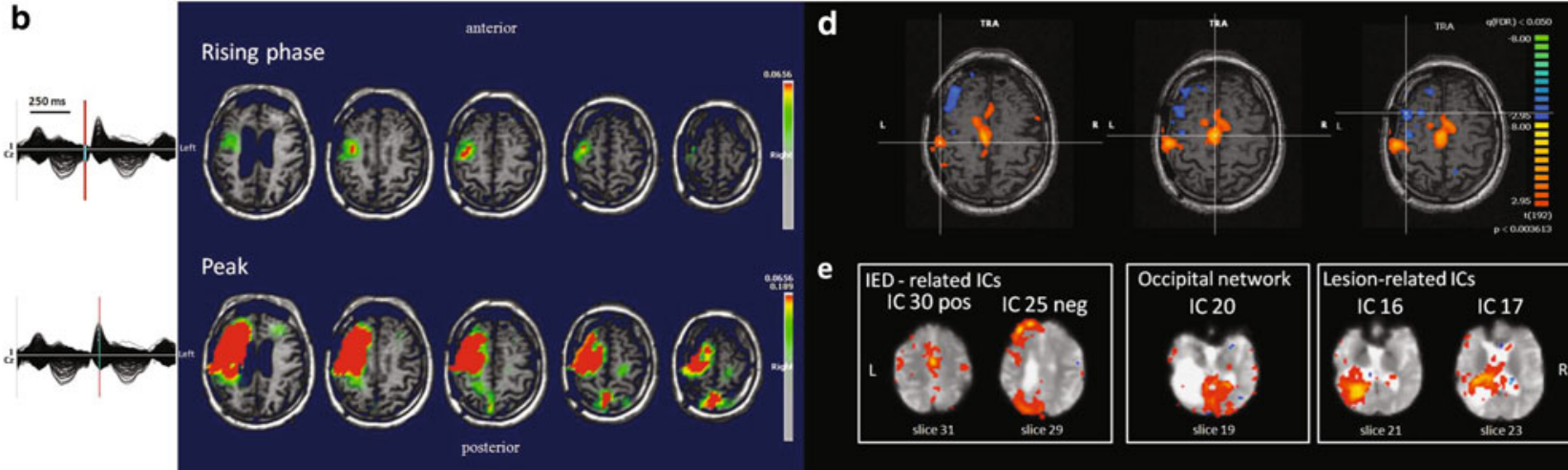

e IED - related ICS
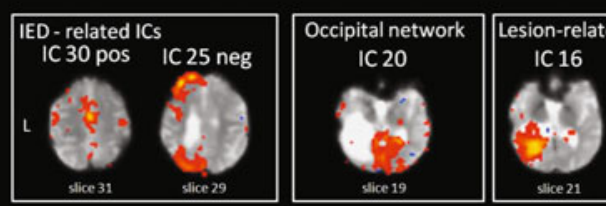

IC 20
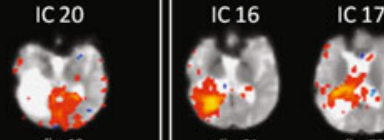

Fig. 1 Patient No. 1. a HdEEG signal visualized according to the projected locations of the scalp electrodes (spike average). b HdEEG source analysis results. On the left, 256-channel EEG traces with a duration of $1 \mathrm{~s}$ (spike average). The global field power is used for the onset (red line). On the right, EEG source imaging in the rising phase of the peak and at the peak. The maximum of the estimated source of

the average interictal spike is indicated in red. $\mathbf{c}$ EEG acquired during fMRI scanning after preprocessing. d EEG-fMRI results from the GLM analysis. Corrected $p$ value (FDR, $p<0.05$ ) is visualized, and the color bar shows the $Z$ score scale. e EEG-fMRI results from the ICA analysis. The maps were thresholded at a posterior probability threshold of $p>0.5$

Patient No. 6 HdEEG was recorded in the waking state and at initial drowsiness. HdEEG showed high activity over the left fronto-central-temporal area (Fig. 6a). The source analysis showed two focal sources near the lesion: one frontal and more anterior and the other more toward the left side (Fig. 6b). EEG inside the magnet showed a slow sequence on the left fronto-temporal electrodes, particularly over F7 (Fig. 6c). EEG-fMRI showed two main changes: the first was characterized by more anterior activation in the frontal area and the second by more lateralized deactivation in the left frontal area, FDR $(q<0.001)$ (Fig. 6d). The PICA decomposition estimated 19 components, five of which were selected: components nos. 1 and 10 were significantly IED-related ICs; the first was negatively and the second positively correlated with the GLM design matrix ( $F$ test, $p<0.05$, corrected for the number of components); component no. 16 was the DMN; components nos. 2 and 3 were lesion-related ICs (Fig. 6e).

Patient No. 7 HdEEG showed maximal amplitude over the right temporal electrodes (Fig. 7a). The source analysis showed a focal source localized in the right temporal lobe

(Fig. 7b). The EEG acquired inside the magnet showed predominant activity over the right temporal regions (T4) (Fig. 7c). According to the GLM analysis, the BOLD activity showed an activation in the right temporal lobe and other sparse activations, FDR $(q<0.05)$ (Fig. 7d). The PICA decomposition estimated 13 components, three of which were selected: the DMN (component no. 9); the occipital network (component no. 1); and the attention network (component no. 2). No IED-related IC was identified (Fig. 7e).

\section{Discussion}

Here, we report on a multimodal approach to the assessment of post-traumatic epilepsy, a complicated pharmacoresistant form of epilepsy. Clinical semeiology, BOLD activation and source localization were compared. Seizure semeiology indicated the onset of seizures; MRI showed the diffuse damage that, although a substrate of post-lesional epilepsy, is not specific enough to localize epileptogenic areas close to or far from injured brain tissue. The 
a

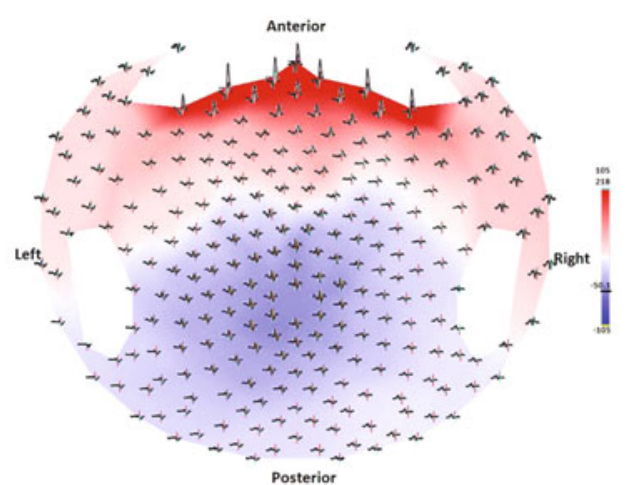

c

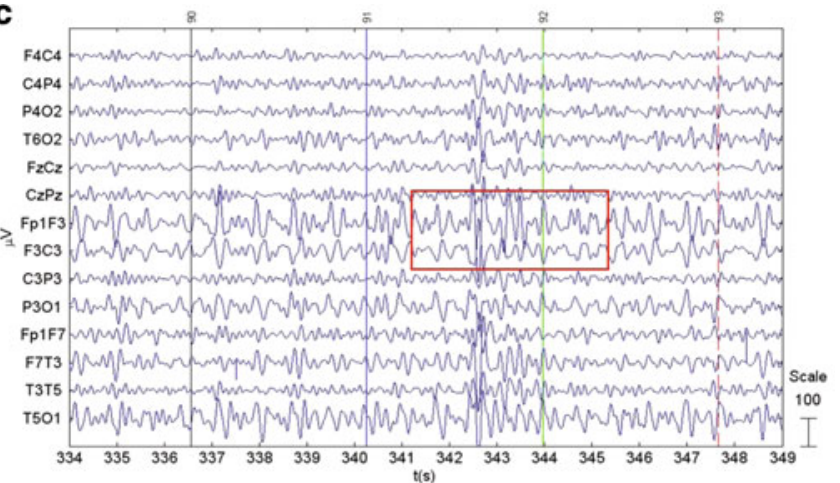

d

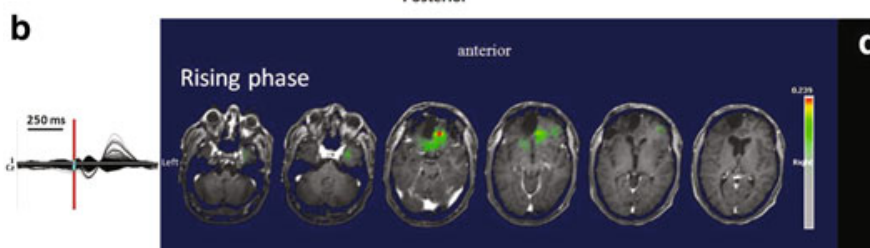

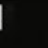
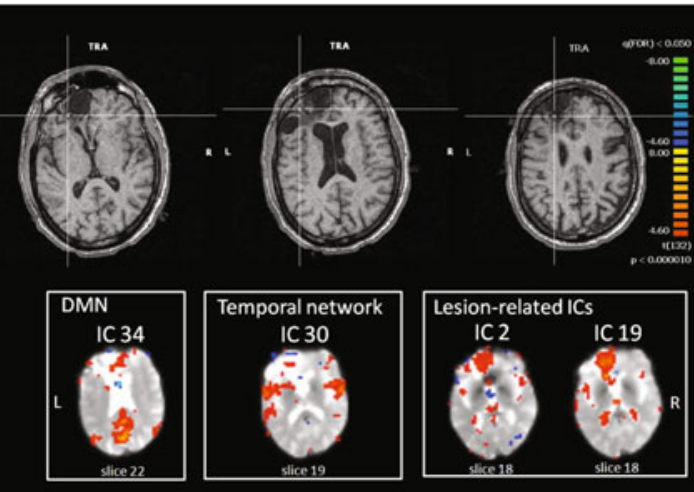

Fig. 2 Patient No. 2. a HdEEG signal visualized according to the projected locations of the scalp electrodes (spike average). b HdEEG source analysis results. On the left, 256-channel EEG traces with a duration of $1 \mathrm{~s}$ (spike average). The global field power is used for the onset (red line). On the right, EEG source imaging in the rising phase of the peak and at the peak. The maximum of the estimated source of

correlation between brain metabolic activity and scalp electrical spikes has been reported in previous studies on different types of epilepsy [57].

The novelty of this study is the use of a multimodal approach in which hdEEG and EEG-fMRI data on pathophysiological mechanisms underlying post-traumatic epilepsy are compared. The utility of a multimodal imaging approach to localize seizures was investigated by studying the relationship between source localization at different time points and BOLD activity. The ESI results showed one or more sources in all seven patients; and in four out of seven cases, the GLM results showed BOLD activation. EEG-fMRI studies demonstrated that IED-related BOLD signal changes can be observed at the same site and remote from the putative seizure onset zone in patients with cortical lesions. While multiple BOLD activations may be interpreted as a measure of network activation in relation to paroxysmal activity, source localization is able to define a focal activity.

Combined recordings as in EEG-fMRI have the dual advantage offered by the high temporal resolution of EEG and the spatial resolution obtained with neuroradiological exams. EEG-fMRI coregistration is widely used to study the average interictal spike is indicated in red. $\mathbf{c}$ EEG acquired during fMRI scanning after preprocessing. d EEG-fMRI results from the GLM analysis. Corrected $p$ value (FDR, $p<0.05$ ) is visualized, and the color bar shows the $Z$ score scale. e EEG-fMRI results from the ICA analysis. The maps were thresholded at a posterior probability threshold of $p>0.5$

the sources of epileptic spikes recorded on the scalp in patients with no visible lesion on MRI [8, 13, 16, 20,44]. Nevertheless, very few data exist on EEG-fMRI coregistration in post-traumatic epilepsy. Baudewig et al. [5] the first to study a patient with generalized epilepsy after head injury, reported BOLD responses compatible with the clinical picture. Rodionov et al. compared the findings of ICA and EEG-based GLM analyses of fMRI data from eight patients with focal epilepsy. In one patient with posttraumatic epilepsy, a single matching independent component (IC) was found to correspond excellently with the GLM result [46]. More recently, Cosottini et al. studied IED-related BOLD response in a patient with post-traumatic focal epilepsy at repeated EEG-fMRI exams. They found that the BOLD response may be focal or multifocal, depending on the spatial distribution of interictal EEG activity, and that the selection of IED within a well-defined focal EEG field, against bilateral diffuse events, can be helpful in the localization of irritative zones and in improving the reproducibility of results [12].

There are limitations to the technique, however. First of all, there are technical problems associated with the interference between the EEG and MR instruments. During 

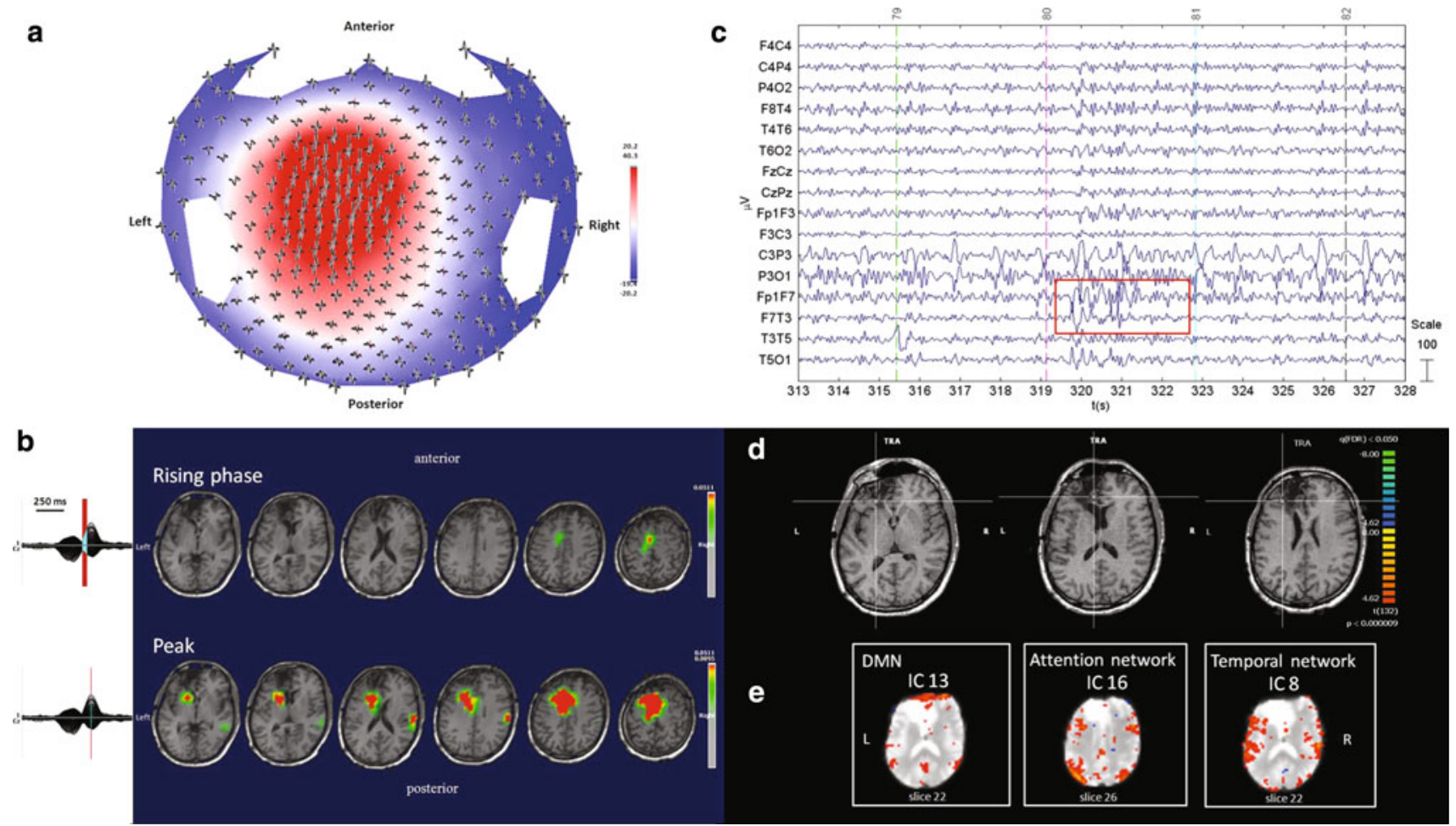

Fig. 3 Patient No. 3. a HdEEG signal visualized according to the projected locations of the scalp electrodes (spike average). b HdEEG source analysis results. On the left, 256-channel EEG traces with a duration of $1 \mathrm{~s} \mathrm{(spike} \mathrm{average).} \mathrm{The} \mathrm{global} \mathrm{field} \mathrm{power} \mathrm{is} \mathrm{used} \mathrm{for} \mathrm{the}$ onset (red line). On the right, EEG source imaging in the rising phase of the peak and at the peak. The maximum of the estimated source of

EEG-fMRI acquired simultaneously, EEG is obscured by artifacts induced by both the static and the time-variant fields of the MR scanner. Simultaneous acquisition introduces common artifact on MR images and on EEG. The MR artifacts on images are caused by electrodes, wires and the EEG system inside the magnet room near the scanner. Differently, the EEG artifacts are caused by movement of the leads themselves within the static filed of the magnet that induces an electromotive force in a wire loop (ballistocardiogram artifact). In addition, the radio-frequency pulses and the gradient switching, required for MRI, may induce voltages that obscure the EEG signal (gradient artifact). These common artifacts on MR images and on EEG can be filtered off-line [18].

Other limitations are related to the epilepsy. First, because it is impossible to predict when epileptic discharges will occur, few IEDs will usually appear during the few minutes of fMRI recording; this hampers the possibility to detect BOLD signal changes [48], e.g., in patients Nos. 2, 3 and 5. Second, because of the volume conductor, deep epileptic discharge cannot be captured by scalp EEG [46]. Nonetheless, these limitations can be overcome by applying multimodal and comparative analysis approaches. the average interictal spike is indicated in red.c EEG acquired during fMRI scanning after preprocessing. d EEG-fMRI results from the GLM analysis. Corrected $p$ value (FDR, $p<0.05$ ) is visualized, and the color bar shows the $Z$ score scale. e EEG-fMRI results from the ICA analysis. The maps were thresholded at a posterior probability threshold of $p>0.5$

In epilepsy evaluation, the most common method to analyze EEG-fMRI data is with the GLM, wherein an a priori hypothesis is made based on the shape of the HRF. With the GLM approach to the evaluation of epileptic patients in resting state, a significant concordance can be observed between focal EEG interictal slow-wave discharges and focal BOLD activation on fMRI.

Different approaches to identifying patterns of coherent activity are used in the analysis of RSNs (for a review see [10]). A method for hypothesis-driven analysis is based on correlating the time course in a certain seed region with the time courses of all other brain voxels. Seed voxel definition, however, is typically based on a priori knowledge of functional localization. Moreover, data-driven analyses have attracted increasing attention: ICA [36]; hierarchical clustering [11]; and Laplacian clustering [52], none of which require a specific prior model definition. In particular, ICA can extract IED-related BOLD components without constraints on the HRF. With ICA, fMRI can be analyzed in the absence of spatial bounds or a priori knowledge about the time course of activation of the different components, whether a component is activated by 
a

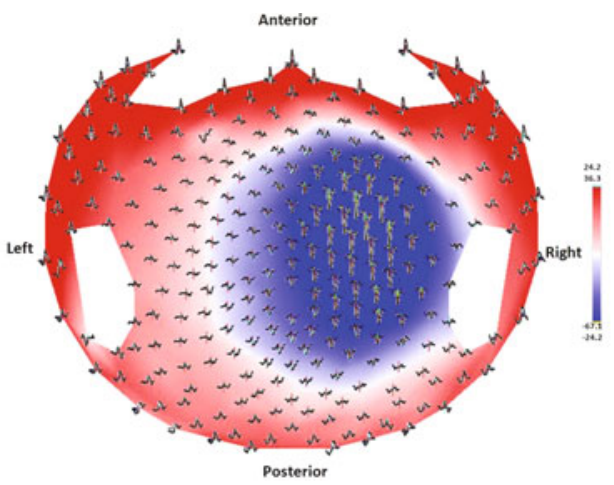

C

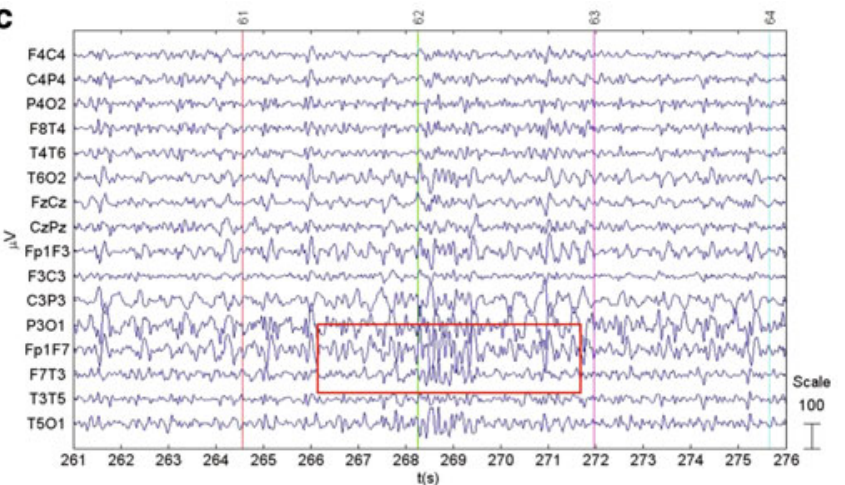

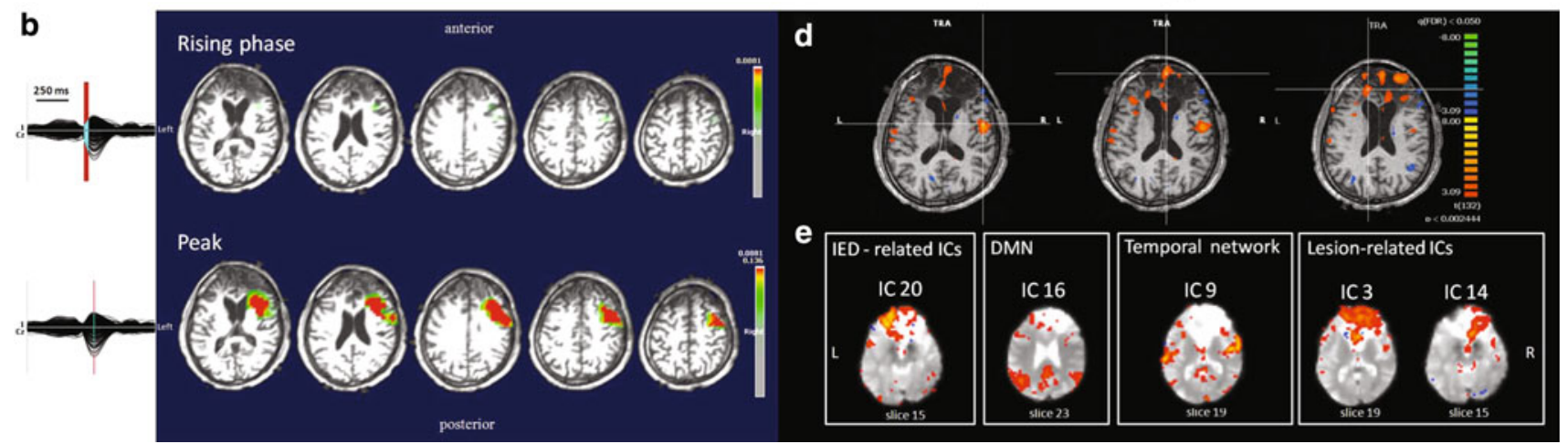

Fig. 4 Patient No. 4. a HdEEG signal visualized according to the projected locations of the scalp electrodes (spike average). b HdEEG source analysis results. On the left, 256-channel EEG traces with a duration of $1 \mathrm{~s}$ (spike average). The global field power is used for the onset (red line). On the right, EEG source imaging in the rising phase of the peak and at the peak. The maximum of the estimated source of the average interictal spike is indicated in red. $\mathbf{c}$ EEG acquired during fMRI scanning after preprocessing. d EEG-fMRI results from the GLM analysis. Corrected $p$ value (FDR, $p<0.05$ ) is visualized, and the color bar shows the $Z$ score scale. e EEG-fMRI results from the ICA analysis. The maps were thresholded at a posterior probability threshold of $p>0.5$

network. The DMN is relevant for two reasons: first, the regions involved in the DMN have dense white matter connections, suggesting that they form part of the brain's structural core. Disruption of the DMN is clinically important, and abnormalities are observed in various neurological and psychiatric disorders [21]. Second, according to published data, the default mode is present as a baseline and is suspended during IED activity [28, 54]. Of note is that DMN was not detected in two patients (patients Nos. 1 and 5) and, when present, it showed modified activation, especially in the frontal lobe (patients Nos. 2, 3, 4, and 6). Patient No. 7, who has temporal focus epilepsy, showed a DMN with very clear frontal activation. A possible explanation is that the brain damage or long-term epileptic activity recorded inside the magnet does not allow activation (or at least in part) of the DMN.

With the advent of simultaneous EEG and fMRI coregistration, complex transient hemodynamic responses associated with ictal and interictal events can be mapped noninvasively. Nevertheless, in patients with epilepsy, EEG remains the mainstay method to investigate functional brain abnormalities and to reveal characteristic paroxystic events reflecting neuronal synchronization. A caveat to this 
a

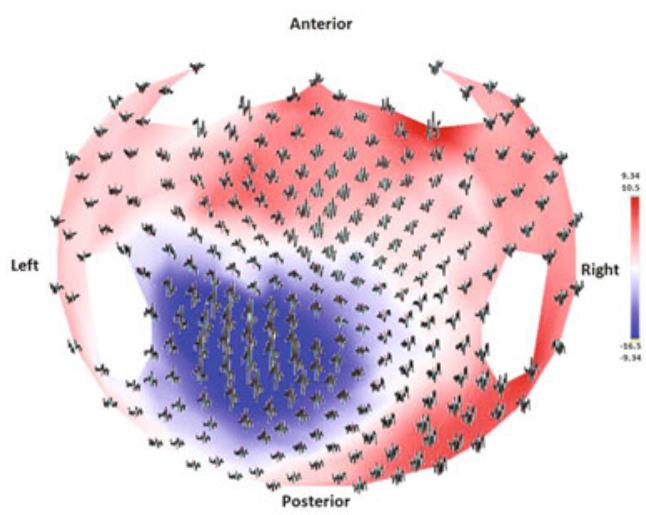

C

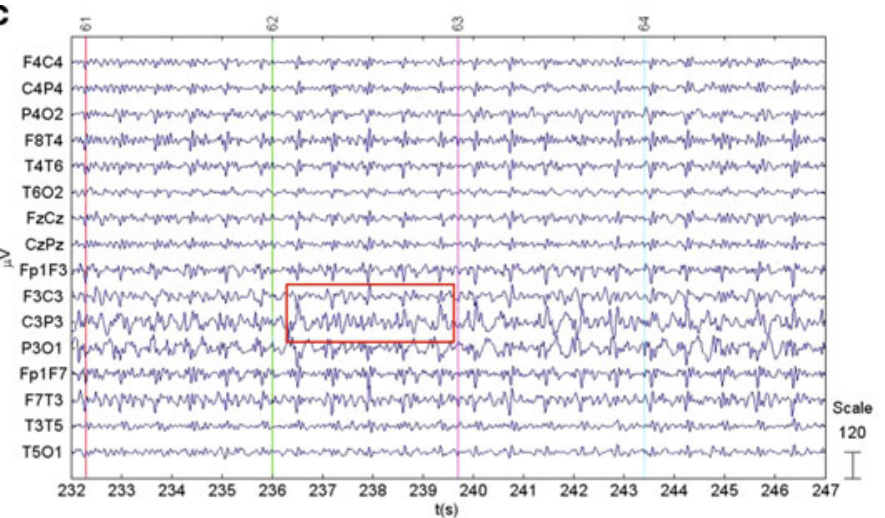

d
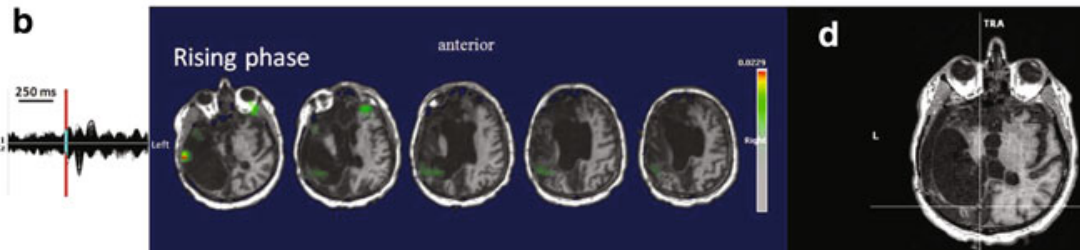

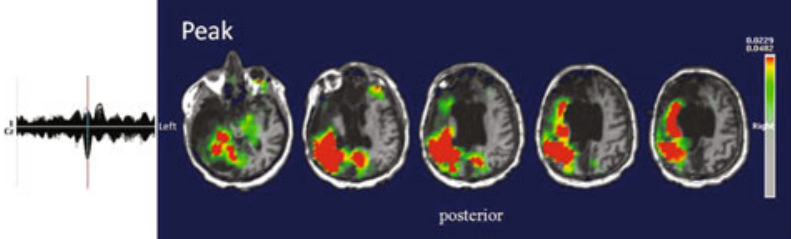

Fig. 5 Patient No. 5. a HdEEG signal visualized according to the projected locations of the scalp electrodes (spike average). b HdEEG source analysis results. On the left, 256-channel EEG traces with a duration of $1 \mathrm{~s} \mathrm{(spike} \mathrm{average).} \mathrm{The} \mathrm{global} \mathrm{field} \mathrm{power} \mathrm{is} \mathrm{used} \mathrm{for} \mathrm{the}$ onset (red line). On the right, EEG source imaging in the rising phase of the peak and at the peak. The maximum of the estimated source of

is that a wrong model of individual geometry and conductivity of the brain and a limited number of EEG channels can influence estimation of the sources. HdEEG technologies were developed to enhance the poor spatial information content of EEG activity. In addition, constraining the source analysis of high spatial resolution EEG to a patient's MRI provides more accurate localization of epileptic activity. The source localization on hdEEG data allows for precise mapping of IED activity when the same analysis on standard EEG fails. Furthermore, the sensitivity of inter-ictal or ictal EEG can be significantly increased with the use of additional zygomatic and pre-frontal electrodes.

The main objective of EEG-fMRI and hdEEG, as an independent electrophysiological and functional measurement, is to view brain function from various different perspectives and to detect and describe changes in neuronal activity. While IED recording and analysis of events can play a central role in the correct diagnosis of epilepsy, simple partial seizures may not have a definite location on scalp EEG. From EEG-fMRI and hdEEG registration, insights can be gained into networks associated with the average interictal spike is indicated in red. c EEG acquired during fMRI scanning after preprocessing. d EEG-fMRI results from the GLM analysis. Corrected $p$ value (FDR, $p<0.05$ ) is visualized, and the color bar shows the $Z$ score scale. e EEG-fMRI results from the ICA analysis. The maps were thresholded at a posterior probability threshold of $p>0.5$

interictal epileptic activity [20]; moreover, having two different methods to localize the same epileptic foci is clinically useful. ESI and simultaneous EEG-fMRI are complementary methods for exploring epileptic activity. fMRI shows a complex network of focal hemodynamic changes related to interictal activity with high spatial resolution, whereas ESI identifies the source based on the electrical scalp activity and reveal the temporal time course of the regions involved during the spike event. The application of multiple methods to investigate the same events is an additional tool which can add sensitivity to the study. In particular, these post-traumatic epileptic patients showed several BOLD activations close to and far from the lesion. Part of these BOLD activations overlapped with the source activity in some cases but not in others, suggesting a more systemic activation or spread activations.

An area where EEG and fMRI integration and ESI have considerable clinical relevance is in the presurgical evaluation of epileptic patients: in many patients with drugresistant focal seizures undergoing epilepsy surgery, standard magnetic resonance imaging scans fail to visualize a clear epileptic source, making preliminary invasive stereo 
a

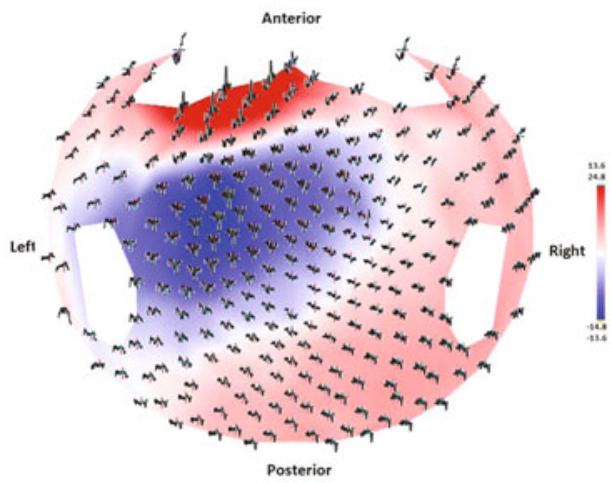

C
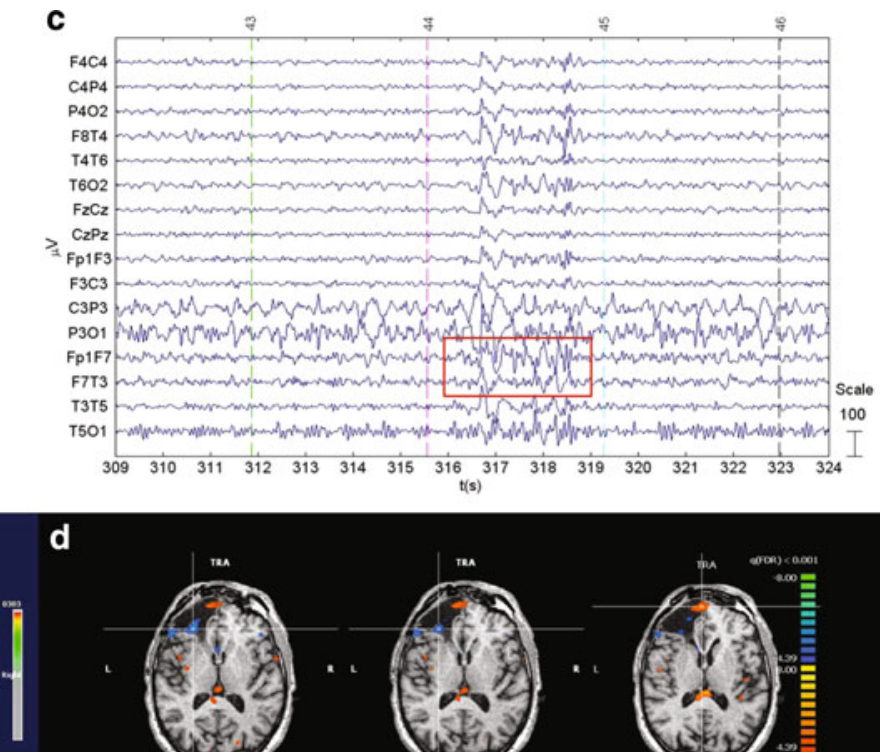

e

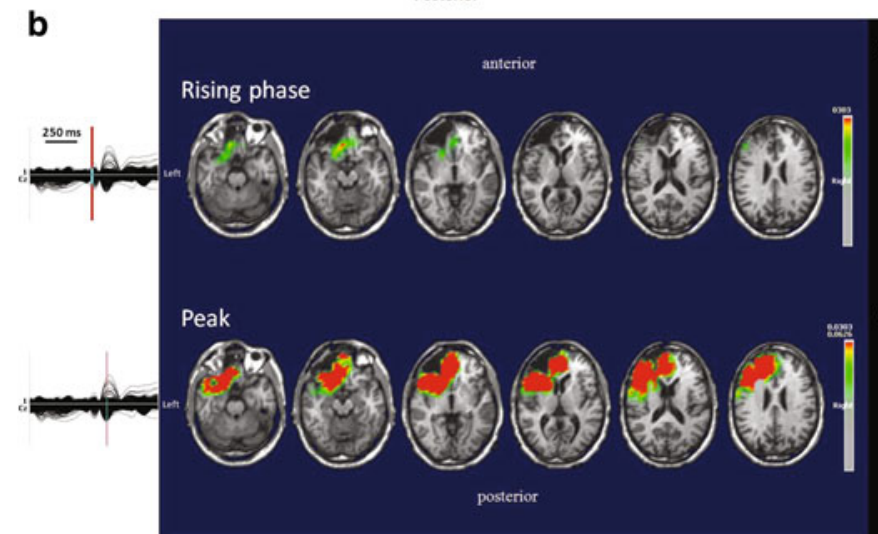

Fig. 6 Patient No. 6. a HdEEG signal visualized according to the projected locations of the scalp electrodes (spike average). b HdEEG source analysis results. On the left, 256-channel EEG traces with a duration of $1 \mathrm{~s}$ (spike average). The global field power is used for the onset (red line). On the right, EEG source imaging in the rising phase of the peak and at the peak. The maximum of the estimated source of

the average interictal spike is indicated in red.c EEG acquired during fMRI scanning after preprocessing. d EEG-fMRI results from the GLM analysis. Corrected $p$ value (FDR, $p<0.001$ ) is visualized, and the color bar shows the $Z$ score scale. e EEG-fMRI results from the ICA analysis. The maps were thresholded at a posterior probability threshold of $p>0.5$

EEG analysis necessary. Simultaneous EEG and fMRI recording and hdEEG offer a less invasive alternative, since they provide valuable information to localize the brain regions generating interictal epileptiform activity. In this group of TBI patients, who are not candidates for any surgical treatment, more information on the areas involved in the epileptic process are necessary in order to understand the physiopathological mechanisms.

On comparison of the hemodynamic and electrophysiological data, the results demonstrated a good correspondence in three out of seven patients (patients Nos. 1, 6, and 7), in three patients (patients Nos. 2, 3 and 5) no BOLD activation was found and in one patient (No. 4) the results were only partially related. The BOLD signal reflects the effect of electrical activity at the microvascular level, with high errors of measure (low signal-to-noise ratio), whereas imprecision in EEG source localization is related to an illposed nature of the problem, to errors of the head and source models. Other possible explanations may be sought in deep epileptic activities recognized by fMRI but not measured on scalp electrodes because of attenuation phenomena. Conversely, asynchronous neurons, which produce only minimal metabolic activities, can be measured by fMRI and not by EEG [45]. In other cases, the two signals can be negatively correlated. A high current density detected by the source localization is associated with hypohemodynamic activity in the same area.

Complementary information can be gleaned from a strategy that combines source analysis and coregistration, particularly in those forms of epilepsy in which clear focal activity is difficult to identify. A multimodal approach could be ideal for assessing patients with post-TBI epilepsy probably because of the long period of spiking alternating with rest periods and because patients with brain injury have sensitive metabolic changes in perilesional tissues. In such patients we cannot exclude changes in perfusion during interictal period due to the functional and anatomical modifications of vessels in a injured brain [35]. In addition the post-traumatic brain has phenomena of maladaptive plasticity due to fiber rearrangement after diffuse axonal injury that can be responsible for the epileptogenesis $[53,55]$. 
a

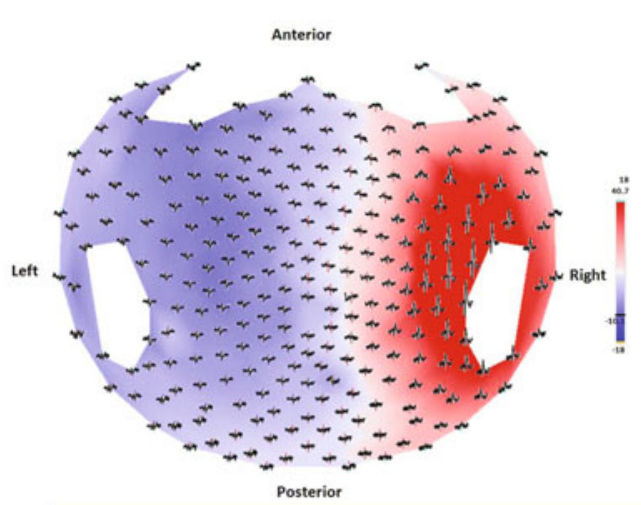

C

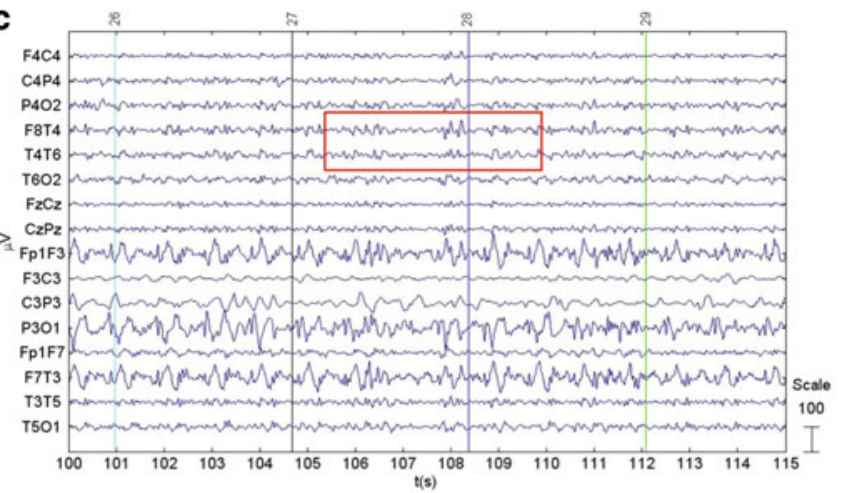

d

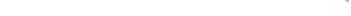

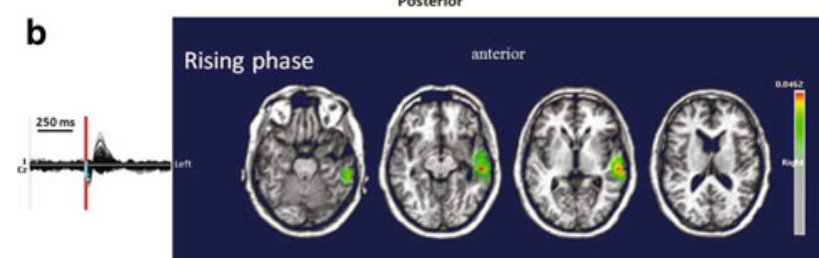

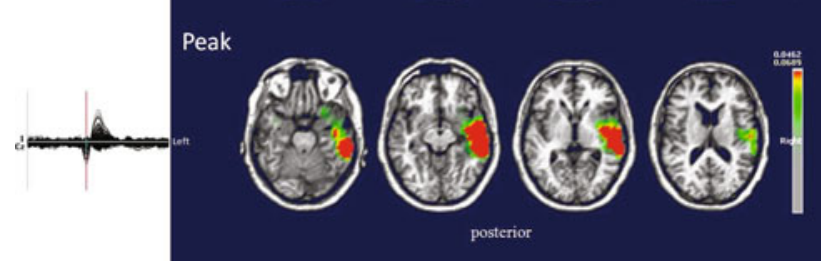

e
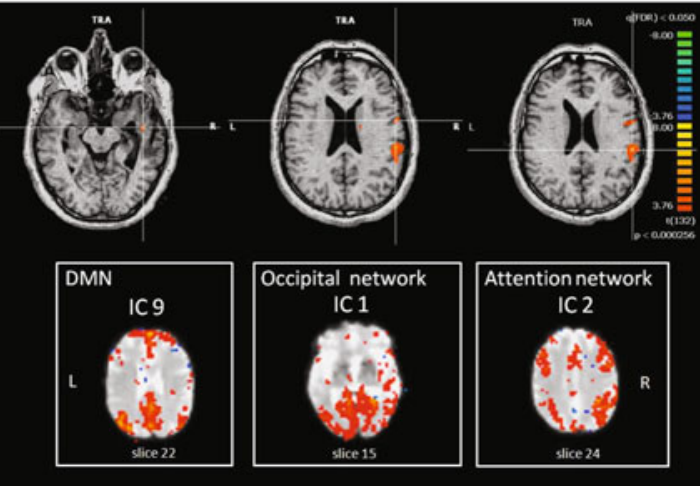

Fig. 7 Patient No. 7. a HdEEG signal visualized according to the projected locations of the scalp electrodes (spike average). b HdEEG source analysis results. On the left, 256-channel EEG traces with a duration of $1 \mathrm{~s}$ (spike average). The global field power is used for the onset (red line). On the right, EEG source imaging in the rising phase of the peak and at the peak. The maximum of the estimated source of

the average interictal spike is indicated in red. $\mathbf{c}$ EEG acquired during fMRI scanning after preprocessing. d EEG-fMRI results from the GLM analysis. Corrected $p$ value (FDR, $p<0.05$ ) is visualized, and the color bar shows the $Z$ score scale. e EEG-fMRI results from the ICA analysis. The maps were thresholded at a posterior probability threshold of $p>0.5$

\section{Conclusion}

Noninvasive imaging techniques such as EEG-fMRI or hdEEG, separately considered, are widely used to investigate abnormal neural activity in relation to BOLD activity and electrical activity, respectively. EEG-fMRI can provide information on the pathophysiological processes underlying interictal activity, since the hemodynamic changes are a consequence of the abnormal neural activity generating IEDs; the source analysis estimates the current density of the source that generates a measured electric potential and it yields a plausible dipole localization of irritative regions. Furthermore, the combined use of EEG-fMRI and hdEEG offers a new and more complete approach to the study of epilepsy and may play an increasingly important role in the evaluation of patients with post-traumatic injury.

Acknowledgments The development of CARTOOL is supported by the Center for Biomedical Imaging (CIBM) of the Universities of Geneva and Lausanne, the Swiss Federal Institute of Technology, Lausanne (EPFL), and the University Hospitals of Geneva and Lausanne, Switzerland. This study was supported by Cariverona, Verona, Italy.

Open Access This article is distributed under the terms of the Creative Commons Attribution License which permits any use, distribution, and reproduction in any medium, provided the original author(s) and the source are credited.

\section{References}

1. Aghakhani Y, Bagshaw AP, Benar CG, Hawco C, Andermann F, Dubeau F, Gotman J (2004) fMRI activation during spike and wave discharges in idiopathic generalized epilepsy. Brain 127:1127-1144

2. Allen PJ, Polizzi G, Krakow K, Fish DR, Lemieux L (1998) Identification of EEG events in the MR scanner: the problem of pulse artifact and a method for its subtraction. Neuroimage 8:229-239

3. Allen PJ, Josephs O, Turner R (2000) A method for removing imaging artifact from continuous EEG recorded during functional MRI. Neuroimage 12:230-239

4. Archer JS, Abbott DF, Waites AB, Jackson GD (2003) fMRI "deactivation" of the posterior cingulate during generalized spike and wave. Neuroimage 20:1915-1922

5. Baudewig J, Bittermann HJ, Paulus W, Frahm J (2001) Simultaneous EEG and functional MRI of epileptic activity: a case report. Clin Neurophysiol 112:1196-1200 
6. Beckmann CF, Smith SM (2004) Probabilistic independent component analysis for functional magnetic resonance imaging. IEEE Trans Med Imaging 23:137-152

7. Benar CG, Gross DW, Wang Y, Petre V, Pike B, Dubeau F, Gotman J (2002) The BOLD response to interictal epileptiform discharges. Neuroimage 17:1182-1192

8. Borelli P, Avesani M, Formaggio E, Storti SF, Zanoni T, Moretto G, Fiaschi A, Cerini R, Manganotti P (2010) EEG-fMRI as an useful tool to detect epileptic foci associated with secondary bilateral synchrony. Seizure 19:605-608

9. Brodbeck V, Spinelli L, Lascano AM, Wissmeier M, Vargas MI, Vulliemoz S, Pollo C, Schaller K, Michel CM, Seeck M (2011) Electroencephalographic source imaging: a prospective study of 152 operated epileptic patients. Brain 134:2887-2897

10. Cole DM, Smith SM, Beckmann CF (2010) Advances and pitfalls in the analysis and interpretation of resting-state FMRI data. Front Syst Neurosci 4:8

11. Cordes D, Haughton V, Carew JD, Arfanakis K, Maravilla K (2002) Hierarchical clustering to measure connectivity in fMRI resting-state data. Magn Reson Imaging 20:305-317

12. Cosottini M, Pesaresi I, Maritato P, Belmonte G, Taddei A, Sartucci F, Mascalchi M, Murri L (2010) EEG topography-specific BOLD changes: a continuous EEG-fMRI study in a patient with focal epilepsy. Magn Reson Imaging 28:388-393

13. Del Felice A, Zanoni T, Avesani M, Formaggio E, Storti S, Fiaschi A, Moretto G, Manganotti P (2009) EEG-fMRI coregistration in non-ketotic hyperglycemic occipital seizures. Epilepsy Res 85:321-324

14. Delorme A, Makeig S (2004) EEGLAB: an open source toolbox for analysis of single-trial EEG dynamics including independent component analysis. J Neurosci Methods 134:9-21

15. Formaggio E, Storti SF, Avesani M, Cerini R, Milanese F, Gasparini A, Acler M, Pozzi Mucelli R, Fiaschi A, Manganotti P (2008) EEG and FMRI coregistration to investigate the cortical oscillatory activities during finger movement. Brain Topogr 21:100-111

16. Formaggio E, Storti SF, Bertoldo A, Manganotti P, Fiaschi A, Toffolo GM (2011) Integrating EEG and fMRI in epilepsy. Neuroimage 54:2719-2731

17. Friston K, Holmes A, Worsley K, Poline JP, Frith C, Frackowiak R (1995) Statistical parametric maps in functional imaging: a general linear approach. Hum Brain Mapp 2:189-210

18. Goldman RI, Stern JM, Engel J, Cohen MS (2000) Acquiring simultaneous EEG and functional MRI. Clin Neurophysiol 111:1974-1980

19. Gotman J, Grova C, Bagshaw A, Kobayashi E, Aghakhani Y, Dubeau F (2005) Generalized epileptic discharges show thalamocortical activation and suspension of the default state of the brain. Proc Natl Acad Sci USA 102:15236-15240

20. Gotman J, Kobayashi E, Bagshaw AP, Benar CG, Dubeau F (2006) Combining EEG and fMRI: a multimodal tool for epilepsy research. J Magn Reson Imaging 23:906-920

21. Greicius M (2008) Resting-state functional connectivity in neuropsychiatric disorders. Curr Opin Neurol 21:424-430

22. Groening K, Brodbeck V, Moeller F, Wolff S, van Baalen A, Michel CM, Jansen O, Boor R, Wiegand G, Stephani U, Siniatchkin M (2009) Combination of EEG-fMRI and EEG source analysis improves interpretation of spike-associated activation networks in paediatric pharmacoresistant focal epilepsies. Neuroimage 46:827-833

23. Guggisberg AG, Dalal SS, Zumer JM, Wong DD, Dubovik S, Michel CM, Schnider A (2011) Localization of cortico-peripheral coherence with electroencephalography. Neuroimage 57:1348-1357

24. Ives JR, Warach S, Schmitt F, Edelman RR, Schomer DL (1993) Monitoring the patient's EEG during echo planar MRI. Electroencephalogr Clin Neurophysiol 87:417-420
25. Jacobs J, Kobayashi E, Boor R, Muhle H, Stephan W, Hawco C, Dubeau F, Jansen O, Stephani U, Gotman J, Siniatchkin M (2007) Hemodynamic responses to interictal epileptiform discharges in children with symptomatic epilepsy. Epilepsia 48:2068-2078

26. Kobayashi E, Bagshaw AP, Grova C, Gotman J, Dubeau F (2006) Grey matter heterotopia: what EEG-fMRI can tell us about epileptogenicity of neuronal migration disorders. Brain 129:366-374

27. Lantz G, Spinelli L, Seeck M, de Peralta Menendez RG, Sottas CC, Michel CM (2003) Propagation of interictal epileptiform activity can lead to erroneous source localizations: a 128-channel EEG mapping study. J Clin Neurophysiol 20:311-319

28. Laufs H, Hamandi K, Salek-Haddadi A, Kleinschmidt AK, Duncan JS, Lemieux L (2007) Temporal lobe interictal epileptic discharges affect cerebral activity in "default mode" brain regions. Hum Brain Mapp 28:1023-1032

29. Lemieux L, Krakow K, Fish DR (2001) Comparison of spiketriggered functional MRI BOLD activation and EEG dipole model localization. Neuroimage 14:1097-1104

30. Lemieux L, Salek-Haddadi A, Josephs O, Allen P, Toms N, Scott C, Krakow K, Turner R, Fish DR (2001) Event-related fMRI with simultaneous and continuous EEG: description of the method and initial case report. Neuroimage 14:780-787

31. Li Q, Luo C, Yang T, Yao Z, He L, Liu L, Xu H, Gong Q, Yao D, Zhou D (2009) EEG-fMRI study on the interictal and ictal generalized spike-wave discharges in patients with childhood absence epilepsy. Epilepsy Res 87:160-168

32. Logothetis NK, Pauls J, Augath M, Trinath T, Oeltermann A (2001) Neurophysiological investigation of the basis of the fMRI signal. Nature 412:150-157

33. Luo C, Yao Z, Li Q, Lei X, Zhou D, Qin Y, Xia Y, Lai Y, Gong Q, Yao D (2010) Imaging foci of epileptic discharges from simultaneous EEG and fMRI using the canonical HRF. Epilepsy Res 91:133-142

34. Manganotti P, Formaggio E, Gasparini A, Cerini R, Bongiovanni LG, Storti SF, Mucelli RP, Fiaschi A, Avesani M (2008) Continuous EEG-fMRI in patients with partial epilepsy and focal interictal slow-wave discharges on EEG. Magn Reson Imaging 26:1089-1100

35. Mazzini L, Cossa FM, Angelino E, Campini R, Pastore I, Monaco F (2003) Posttraumatic epilepsy: neuroradiologic and neuropsychological assessment of long-term outcome. Epilepsia 44:569-574

36. McKeown MJ, Makeig S, Brown GG, Jung TP, Kindermann SS, Bell AJ, Sejnowski TJ (1998) Analysis of fMRI data by blind separation into independent spatial components. Hum Brain Mapp 6:160-188

37. Menon V, Crottaz-Herbette S (2005) Combined EEG and fMRI studies of human brain function. Int Rev Neurobiol 66:291-321

38. Michel CM, Murray MM, Lantz G, Gonzalez S, Spinelli L, Gravede Peralta R (2004) EEG source imaging. Clin Neurophysiol 115:2195-2222

39. Moeller F, LeVan P, Gotman J (2011) Independent component analysis (ICA) of generalized spike wave discharges in fMRI: comparison with general linear model-based EEG-fMRI. Hum Brain Mapp 32:209-217

40. Niedermeyer E, Lopesda Silva FH (2004) Electroencephalography: basic principles, clinical applications, and related fields, 5th edn. Lippincott Williams \& Wilkins, Philadelphia

41. Ogawa S, Lee TM, Stepnoski R, Chen W, Zhu XH, Ugurbil K (2000) An approach to probe some neural systems interaction by functional MRI at neural time scale down to milliseconds. Proc Natl Acad Sci USA 97:11026-11031

42. Ossenblok P, Fuchs M, Velis DN, Veltman E, Pijn JP, da Silva FH (1999) Source analysis of lesional frontal-lobe epilepsy. IEEE Eng Med Biol Mag 18:67-77 
43. Pascual-Marqui RD, Michel CM, Lehmann D (1994) Low resolution electromagnetic tomography: a new method for localizing electrical activity in the brain. Int J Psychophysiol 18:49-65

44. Pesaresi I, Cosottini M, Belmonte G, Maritato P, Mascalchi M, Puglioli M, Sartucci F, Bartolozzi C, Murri L (2011) Reproducibility of BOLD localization of interictal activity in patients with focal epilepsy: intrasession and intersession comparisons. Magn Reson Mater Phy 24:285-296

45. Ritter P, Villringer A (2006) Simultaneous EEG-fMRI. Neurosci Biobehav Rev 30:823-838

46. Rodionov R, De Martino F, Laufs H, Carmichael DW, Formisano E, Walker M, Duncan JS, Lemieux L (2007) Independent component analysis of interictal fMRI in focal epilepsy: comparison with general linear model-based EEG-correlated fMRI. Neuroimage 38:488-500

47. Rosenkranz K, Lemieux L (2010) Present and future of simultaneous EEG-fMRI. Magn Reson Mater Phy 23:309-316

48. Salek-Haddadi A, Diehl B, Hamandi K, Merschhemke M, Liston A, Friston K, Duncan JS, Fish DR, Lemieux L (2006) Hemodynamic correlates of epileptiform discharges: an EEG-fMRI study of 63 patients with focal epilepsy. Brain Res 1088:148-166

49. Siniatchkin M, Groening K, Moehring J, Moeller F, Boor R, Brodbeck V, Michel CM, Rodionov R, Lemieux L, Stephani U (2010) Neuronal networks in children with continuous spikes and waves during slow sleep. Brain 133:2798-2813

50. Spinelli L, Andino SG, Lantz G, Seeck M, Michel CM (2000) Electromagnetic inverse solutions in anatomically constrained spherical head models. Brain Topogr 13:115-125

51. Storti SF, Formaggio E, Beltramello A, Fiaschi A, Manganotti P (2010) Wavelet analysis as a tool for investigating movementrelated cortical oscillations in EEG-fMRI coregistration. Brain Topogr 23:46-57
52. Thirion B, Dodel S, Poline JB (2006) Detection of signal synchronizations in resting-state fMRI datasets. Neuroimage 29:321-327

53. Thom M, Liu JY, Thompson P, Phadke R, Narkiewicz M, Martinian L, Marsdon D, Koepp M, Caboclo L, Catarino CB, Sisodiya SM (2011) Neurofibrillary tangle pathology and Braak staging in chronic epilepsy in relation to traumatic brain injury and hippocampal sclerosis: a post-mortem study. Brain 134:2969-2981

54. Vaudano AE, Laufs H, Kiebel SJ, Carmichael DW, Hamandi K, Guye M, Thornton R, Rodionov R, Friston KJ, Duncan JS, Lemieux L (2009) Causal hierarchy within the thalamo-cortical network in spike and wave discharges. PLoS ONE 4:e6475

55. Volman V, Sejnowski TJ, Bazhenov M (2011) Topological basis of epileptogenesis in a model of severe cortical trauma. J Neurophysiol 106:1933-1942

56. Vulliemoz S, Thornton R, Rodionov R, Carmichael DW, Guye M, Lhatoo S, McEvoy AW, Spinelli L, Michel CM, Duncan JS, Lemieux L (2009) The spatio-temporal mapping of epileptic networks: combination of EEG-fMRI and EEG source imaging. Neuroimage 46:834-843

57. Vulliemoz S, Lemieux L, Daunizeau J, Michel CM, Duncan JS (2010) The combination of EEG source imaging and EEG-correlated functional MRI to map epileptic networks. Epilepsia 51:491-505

58. Vulliemoz S, Rodionov R, Carmichael DW, Thornton R, Guye M, Lhatoo SD, Michel CM, Duncan JS, Lemieux L (2010) Continuous EEG source imaging enhances analysis of EEG-fMRI in focal epilepsy. Neuroimage 49:3219-3229 